\title{
Molecular Identification of Resistance Determinants, Integrons and Genetic Relatedness of Extensively Drug Resistant Acinetobacter baumannii Isolated From Hospitals in Tehran, Iran
}

\author{
Shahin Najar Peerayeh ${ }^{1}$; Afsaneh Karmostaji ${ }^{2, *}$ \\ ${ }^{1}$ Department of Bacteriology, Faculty of Medical Sciences, University of Tarbiat Modares, Tehran, IR Iran \\ ${ }^{2}$ Infectious and Tropical Diseases Research Center, Hormozgan University of Medical Sciences, Bandar Abbas, IR Iran \\ *Corresponding author: Afsaneh Karmostaji, Infectious and Tropical Diseases Research Center, Hormozgan University of Medical Sciences, Bandar Abbas, IR Iran. Tel: +98-9177614512, \\ Fax:+98-7636670724, E-mail:Afsanehkk@yahoo.com
}

Received: January 24, 2015; Revised: May 1, 2015; Accepted: May 25, 2015

\begin{abstract}
Background: Acinetobacter baumannii has emerged as an important nosocomial pathogen. Hospital outbreaks of extensively drug resistant(XDR)A. baumannii are a great concern.

Objectives: Aims of this study were to characterize the resistance determinants and genetic relatedness of (XDR) A. baumannii isolates in hospitals in Tehran, Iran.

Materials and Methods: During a three-year study, clinical isolates of A. baumannii were collected from two hospitals in Tehran, Iran. Susceptibility testing to antibiotics was performed by disk diffusion method and XDR A. baumannii isolates were identified. Genes' encoding for carbapenemase production and integrons were identified by PCR. MICs of imipenem and meropenem were determined by agar dilution. Multiple locus variable-number tandem repeat analysis (MLVA) typing was used to determine genetic relationships of XDR isolates.

Results: Using PCR for amplification of bla ${ }_{\text {OXA-51, }}$ 93.9\% (123.131) of isolates were identified as A. baumannii and 24.4\% (30.123) were XDR. These isolates were resistant to gentamicin, ciprofloxacin, amikacin, cotrimoxazole, cefepime, cefotaxime, aztreonam and ceftazidime. Thirty percent of the isolates were resistant to tigecycline. All isolates were susceptible to colistin and polymyxin-B, while 93.3\% (28.30) possessed bla OXA-23 $^{- \text {-like and 6.7\%(2.30) possessed bla }}$ OXA-24-like. All isolates possessed insertion sequence (ISAba1) in the upstream region of the ${ }_{\text {OXA-23 }}$-like gene. Almost 96.7\% (29.30) of the isolates were positive for class I integron and $43.3 \%(13.30)$ for class II. These isolates were also positive for class I. Class III integron was not detected. MLVA typing of XDR isolates showed seven clonally complexes and 16 singletons. Conclusions: The population structure of the A. baumannii isolates in our hospitals was genetically diverse. A significant association between XDR pattern and presence of class 1 integron $(\mathrm{P}<0.001)$ was found indicating that many antibiotic resistance determinants are involved in development of XDR strains.
\end{abstract}

Keywords: Extensively Drug-Resistant; Integrons; Oxacillinase; Acinetobacter baumannii

\section{Background}

Acinetobacter species are emerging as one of the most important nosocomial pathogens that cause a variety of infections in patients, especially among those in intensive care units. These patients are at risk of different infections such as ventilator-associated pneumonia, bacteremia, surgical wound infections, meningitis and urinary tract infections (1). Due to resistance to multiple classes of antibiotics, only a few treatment options are available for carbapenem-resistant Acinetobacter baumannii (CRAB). Genetically mobile elements, including integrons, carry diverse arrays of resistant gene cassettes and promote the dissemination of resistance determinants (2). The transfer of integrons carrying resistance gene cassettes to other bacteria results in the emergence of resistance to multiple drugs (3). Acquired carbapenem resistance in Acinetobacter is often associated with acquired carbapen- emase production, including OXA-23, OXA-24 and OXA-58 type class D carbapenemases (1).

The insertion sequence ISAba1 has been found to be of concern with several antibiotic-resistant genes, including $b l a_{\mathrm{OXA}-23}, b l a_{\mathrm{OXA}-58}$ and bla ${ }_{\mathrm{OXA}-51}$, and it appears to provide the promoter required for gene expression (4). A strain that is resistant to at least three classes of antimicrobial agents, i.e. all 1) penicillins and cephalosporins (including inhibitor combinations), 2) fluoroquinolones and 3) aminoglycosides, is referred to as being multi-drug resistant (MDR). An MDR strain that is also resistant to imipenem is referred to as extensively drug resistant (XDR) (5). Major epidemiological features of this organism are its propensity for being spread clonally (6). MlVA typing of A. baumannii with eight variable number tandem repeat (MLVA-8), had been optimized

Copyright (C) 2015, Ahvaz Jundishapur University of Medical Sciences. This is an open-access article distributed under the terms of the Creative Commons Attribution-NonCommercial 4.0 International License (http://creativecommons.org/licenses/by-nc/4.0/) which permits copy and redistribute the material just in noncommercial usages, provided the original work is properly cited. 
by Pourcel et al. and compared with typing methods, including PFGE and SG profiling (7).

\section{Objectives}

Considering the paucity of epidemiological information on the genetic structure and prevalence of resistant genes in Iran, in this study, we applied MLVA- 8 to the typing of an extensively drug-resistant $A$. baumannii isolates collected at two different hospitals in Tehran, Iran. We also evaluated the occurrence of OXA-like carbapenemase and integrons among XDR strains.

\section{Materials and Methods}

\subsection{Bacterial Isolates}

The study was conducted from March 2010 to November 2013 in two hospitals in Tehran, Iran. Acinetobacter was identified by conventional testing methods. One hundred and thirty one isolates were collected from specimens of affected patients, (e.g., sputum, urine, cerebrospinal fluid and pleural effusion).

\subsection{Susceptibility Testing}

Susceptibility testing to antibiotics, including tigecycline, colistin and other antimicrobial agents, was performed by disk diffusion method as recommended by the clinical and laboratory standards institute (CLSI) (8). Tigecycline (TGC $15 \mu \mathrm{g})$, colistin (CO $10 \mu \mathrm{g}$ ), imipenem (IMI $10 \mu \mathrm{g})$, meropenem (MEN $10 \mu \mathrm{g})$, gentamicin (GM $10 \mu \mathrm{g}$ ), ciprofloxacin (CIP $5 \mu \mathrm{g}$ ), Amikacin (AK 30 $\mu \mathrm{g}$ ), cotrimoxazole (TS $25 \mu \mathrm{g}$ ), cefepime (CPM30 $\mu \mathrm{g}$ ), cefotaxime (CTX $30 \mu \mathrm{g})$, aztreonam (ATM $30 \mu \mathrm{g})$, ceftazidime (CAZ $30 \mu \mathrm{g})$ and polymyxin B (PB $300 \mathrm{U}$ ) were obtained from MAST Pharmaceuticals, Inc. UK. Quality control was performed by testing the susceptibility of Escherichia coli ATCC 25922.

\subsection{PCR Amplification}

DNA was extracted from the isolates by boiling method (9). All isolates were subjected to multiplex PCR to detect the bla $O X A-51^{-l i k e}$ gene that is unique to A. baumannii species $(10,11)$ and to detect the genes of the carbapenemhydrolyzing oxacillinases OXA-23, OXA-58 and OXA-24 (12). PCR primers and the annealing temperature are listed in Table 1. Class 1, 2 and 3 integrons were detected using multiplex PCR, as described by Dillon et al. (13). A. baumannii strain ATCC 17978 and referenced strain COL 20820 was used as positive control for phenotypic tests and detection of bla OXA-51.

\subsection{Molecular Typing}

MLVA was used to determine the epidemiological relationship among A. baumannii XDR isolates harboring integrons and carbapenem resistance genes according to Pourcel et al. and the variable number tandem repeat (VNTR) markers used were L-repeats VNTR, including Abaum3530, Abaum3002, Abaum2240, Abaum1988 and S-repeats VNTR, including Abaum0826, Abaum2396, Abaum3468 and Abaum0845 (7). The polymorphism indices of individual loci or combined VNTR loci and confidence intervals (CIs) were calculated using the Hunter-Gaston diversity index (HGDI) by hpa bioinformatic on line tools software $(14,15)$. The length of repeat, the number of repetitions and deduced sizes of the flanking regions were analyzed (16). Cluster analysis of the MLVA typing data was performed by MLVA plus online tools software.

\begin{tabular}{|c|c|c|c|}
\hline Gene & Primer & Amplicon Size & Annealing \\
\hline blaOXA-23F & GAT CGG ATT GGA GAA CCAGA & \multirow[t]{2}{*}{$501 \mathrm{bp}$} & \multirow[t]{2}{*}{53} \\
\hline blaOXA-23R & ATT TCT GAC CGC ATT TCC AT & & \\
\hline blaOXA-24F & GGT TAG TTG GCC CCC TTA AA & \multirow[t]{2}{*}{246 bp } & \multirow[t]{2}{*}{53} \\
\hline blaOXA-24R & AGT TGA GCG AAA AGG GGA TT & & \\
\hline blaOXA-58F & AAG TAT TGG GGC TTG TGC TG & \multirow[t]{2}{*}{599 bp } & \multirow[t]{2}{*}{53} \\
\hline blaOXA-58R & CCССТCTGCGCTCTACATAC & & \\
\hline Isaba-1(F) & CACGAATGCAGAAGTTG & \multirow[t]{2}{*}{$1200 \mathrm{bp}$} & \multirow[t]{2}{*}{49} \\
\hline OXA-23-R & TTAAATAATATTCAGCTGT & & \\
\hline Int-1F & CAGTGGACATAAGCCTGTTC & \multirow[t]{2}{*}{$160 \mathrm{bp}$} & \multirow[t]{2}{*}{62} \\
\hline Int-1R & CCCGAGGCATAGACTGTA & & \\
\hline Int-2F & GTAGCAAACGAGTGACGAAATG & \multirow[t]{2}{*}{788 bp } & \multirow[t]{2}{*}{62} \\
\hline Int-2R & CACGGATATGCGACAAAAAGGT & & \\
\hline Int-3F & GCCTCCGGCAGCGACTTTCAG & \multirow[t]{2}{*}{979 bp } & \multirow[t]{2}{*}{62} \\
\hline Int-3R & ACGGATCTGCCAAACCTGACT & & \\
\hline
\end{tabular}


Najar Peerayeh S et al.

\section{Results}

Using PCR for amplification of bla $a_{\mathrm{OXA}-51}, 123$ of 131 Acinetobacter isolates were identified as A. baumannii. Thirty isolates (24.4\%) were XDR. According to disc diffusion, these isolates were resistant to imipenem, gentamicin, ciprofloxacin, amikacin, cotrimoxazole, cefepime, cefotaxime, aztreonam and ceftazidime. Thirty percent of these XDR isolates were resistant to tigecycline based on the FDA criteria. All isolates were susceptible to colistin and polymyxin-B. The minimum inhibitory concentration (MICs) of imipenem and meropenem for the $A$. baumannii isolates ranged from 16 to $128 \mathrm{mg} / \mathrm{L}$ and 4 to $256 \mathrm{mg} / \mathrm{L}$, respectively. According to the MICs, 30 (100\%) of XDR A. baumannii isolates were resistant to imipenem and 29 (96.7\%) were resistant to meropenem (MIC $\geq 16$ $\mathrm{mg} / \mathrm{L}$ ). bla OXA-58 gene was not detected in XDR isolates.

Twenty-eight XDR isolates (93.3\%) had positive result for

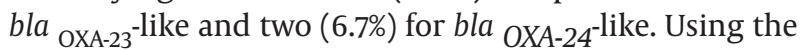
ISAba1 forward primer and the OXA-23-like gene reverse primer, all isolates yielded a PCR amplicon of $1.2 \mathrm{~kb}$, indicating the location of ISAba1 in the upstream region of the OXA-23-like gene of these isolates. Twenty-nine XDR isolates (96.7\%) carrying class 1 integrons and 13 (43.3\%) were carrying both class I and II integrons. Class III integrons were not detected. The MLVA typing of the XDR isolates showed seven clonally complexes and 16 singletons. Some isolates with the same clonally complex and obtained from the same ward, were different in the combination of resistance genes. Figure 1 shows the minimum spanning tree presentation of MLVA-8 clustering of the XDR strains. In our study, Abaum3468 had amplification failure.

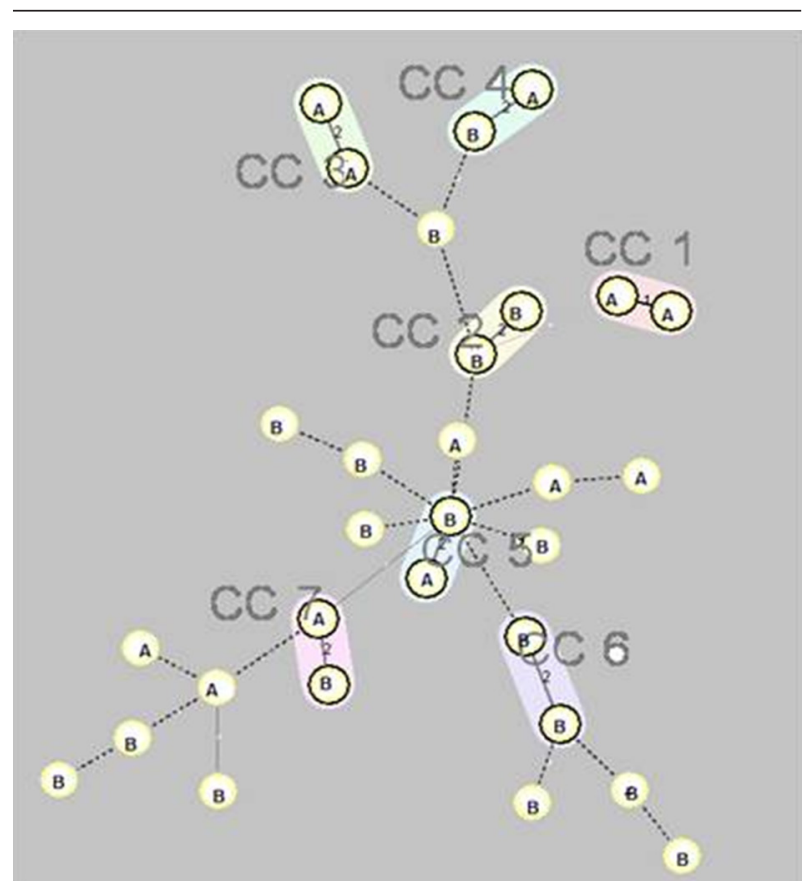

Figure 1. Minimum Spanning Tree Representation of the MLVA-8 Clustering of the 30XDR Isolates
Seven complexes (cc1 to cc7) are designated and marked by different halo colors. A distance of two loci is indicated by a thin line and a distance of three or more loci is indicated by a broken line. The letters inside the circles (A and B) indicate the name of hospitals.

\section{Discussion}

Genotyping XDR isolates by MLVA indicated that different strains were co-circulating within the two hospitals. This is the first population genetic study on A. baumannii by MLVA typing in Iran and we demonstrated that an XDR A. baumannii population in our hospitals was genetically diverse, which complicates preventive and infection control measures. Strain typing in another study also showed genotypic diversity within A. baumannii (17), but still other studies described them clonally $(18,19)$. This phenomenon could be due to the use of three S-repeats VNTR loci with high discriminative power in our study. The four L-repeats, i.e. Abaum3530, Abaum3002, Abaum2240 and Abaum1988, were distributed in the major clones, and the S-repeats, i.e. Abaum0826, Abaum0845 Abaum2396 and Abaum3468 provided a high level of discrimination. The diversity of MLVA S-VNTR markers, led to fine clustering of isolates.

Using pulsed-field gel electrophoresis (PFGE) along with MLVA can provide better tool for epidemiological interpretation that was not used in our study. In a French study, three loci, i.e. Abaum3002, Abaum3530 and Abaum 0826, had amplification failure (20). Nevertheless, in our study Abaum3468 had amplification failure. This reflects the fact that the distribution of the loci in different strains was not uniform. In all of the resistant strains examined, the bla- ${ }_{\text {OXA-23 }}$ gene was along with to the insertion sequence ISAba1. The presence of bla- ${ }^{-}{ }^{-}$in 23 several different MLVA types in different hospitals implies its mobility. Identification of common genetic elements (ISAba1-bla OXA-23 and the integrons) in isolates with different clonal complexes suggested that horizontal transfer occurred rather than clonally spreading of the isolates. Additional studies with longer period of time are needed to assess this phenomenon. In this study, we detected class I and class II integrons in A. baumannii in $96.7 \%$ and $43.3 \%$ of the clinical isolates, respectively.

Among 115 class 1 integron-positive isolates, 57 (49.6\%) isolates were meropenem and imipenem resistant. There was no association between the presence of class 1 integron and resistance to imipenem and meropenem ( $P>0.05)$; however, a significant association between XDR pattern and presence of class 1 integron $(\mathrm{P}<0.001)$ was found. Since class I integrons carrying multiple resistance gene cassettes, association with XDR resistant strains is not unexpected. In an Australian study, a multiplex PCR method targeting the specific integrase genes (intI) for class I, II and III integrons failed to identify intII (encoding the class II integrase) or intIII (class III) in any isolate (4). In Thailand, among 63 MDR A. baumannii, $31(67 \%)$ and $2(4 \%)$ isolates carried class I and class 
II integrons. Class III integrons were not detected. The first report of class I integrons in multidrug-resistant $A$. baumannii in northwest Iran, showed that $92.5 \%$ of MDR A. baumannii carried class I integrons. The presence of class 1 integrons had a significant association with resistance in MDR-A. baumannii (21). Moreover, presence of class I and II integrons in 47 (53.4\%) of MDR A. baumannii isolates was reported in southern Iran, while similar to the present study, class III integron was not detected (22). Our study showed resistance to most of the available antimicrobial agents for the treatment of infections caused by A. baumannii, except for polymyxin-B and colistin. The broad-spectrum in vitro activity of tigecycline and colistin may make them suitable candidates for use in the treatment of XDR A. baumannii.

\section{Acknowledgements}

The study was supported by a grant from faculty of medical sciences, Tarbiat Modares university of Iran.

\section{Authors' Contributions}

Shahin Najar Peerayeh developed the study concept and design, acquisition of data and study supervision, technical and material support. Afsaneh Karmostaji was responsible for drafting of the manuscript, acquisition of data, analysis and interpretation of data and statistical analysis.

\section{Funding/Support}

Financial support and equipment of this research was provided by Tarbiat Modares university.

\section{References}

1. Wang H, Guo P, Sun H, Wang H, Yang Q, Chen M, et al. Molecular epidemiology of clinical isolates of carbapenem-resistant Acinetobacter spp. from Chinese hospitals. Antimicrob Agents Chemother. 2007;51(11):4022-8.

2. Sirichot S, Diraphat P, Utrarachkij F, Tribuddharat C, Siripanichgon K. Dissemination of class I integron in Acinetobacter baumliannii isolated from ventilator-associated pneumonia patients and their environment. Southeast Asian J Trop Med Public Health. 2009;40(6):1284-92.

3. Huang LY, Chen TL, Lu PL, Tsai CA, Cho WL, Chang FY, et al. Dissemination of multidrug-resistant, class 1 integron-carrying Acinetobacter baumannii isolates in Taiwan. Clin Microbiol Infect. 2008;14(11):1010-9.

4. Valenzuela JK, Thomas L, Partridge SR, van der Reijden T, Dijkshoorn L, Iredell J. Horizontal gene transfer in a polyclonal outbreak of carbapenem-resistant Acinetobacter baumannii. J Clin Microbiol. 2007;45(2):453-60.

5. Manchanda V, Sanchaita S, Singh N. Multidrug resistant acinetobacter. J Glob Infect Dis. 2010;2(3):291-304.
6. Hu Y, Li B, Jin D, Cui Z, Tao X, Zhang B, et al. Comparison of multiple-locus variable-number tandem-repeat analysis with pulsedfield gel electrophoresis typing of Acinetobacter baumannii in China. J Clin Microbiol. 2013;51(4):1263-8.

7. Pourcel C, Minandri F, Hauck Y, D'Arezzo S, Imperi F, Vergnaud G, et al. Identification of variable-number tandem-repeat (VNTR) sequences in Acinetobacter baumannii and interlaboratory validation of an optimized multiple-locus VNTR analysis typing scheme. J Clin Microbiol. 2011;49(2):539-48.

8. Clinical and laboratory standards institute . Performance standards for Antimicrobil susceptibility testing; twentieth informational supplement. Wayne 2010.

9. Andriamanantena TS, Ratsima E, Rakotonirina HC, Randrianirina F, Ramparany L, Carod JF, et al. Dissemination of multidrug resistant Acinetobacter baumannii in various hospitals of Antananarivo Madagascar. Ann Clin Microbiol Antimicrob. 2010;9:17.

10. Alsultan AA, Hamouda A, Evans BA, Amyes SG. Acinetobacter baumannii: emergence of four strains with novel bla(OXA51-like) genes in patients with diabetes mellitus. $J$ Chemother. 2009;21(3):290-5.

11. Turton JF, Woodford N, Glover J, Yarde S, Kaufmann ME, Pitt TL Identification of Acinetobacter baumannii by detection of the blaOXA-51-like carbapenemase gene intrinsic to this species. Clin Microbiol. 2006;44(8):2974-6.

12. Woodford N, Ellington MJ, Coelho JM, Turton JF, Ward ME, Brown S, et al. Multiplex PCR for genes encoding prevalent OXA carbapenemases in Acinetobacter spp. Int J Antimicrob Agents. 2006;27(4):351-3.

13. Dillon B, Thomas L, Mohmand G, Zelynski A, Iredell J. Multiplex PCR for screening of integrons in bacterial lysates. J Microbiol Methods. 2005;62(2):221-32.

14. Hunter PR, Gaston MA. Numerical index of the discriminatory ability of typing systems: an application of Simpson's index of diversity.J Clin Microbiol. 1988;26(11):2465-6.

15. Grundmann H, Hori S, Tanner G. Determining confidence intervals when measuring genetic diversity and the discriminatory abilities of typing methods for microorganisms. J Clin Microbiol. 2001;39(11):4190-2.

16. Benson G. Tandem repeats finder: a program to analyze DNA sequences. Nucleic Acids Res. 1999;27(2):573-80.

17. Diancourt L, Passet V, Nemec A, Dijkshoorn L, Brisse S. The population structure of Acinetobacter baumannii: expanding multiresistant clones from an ancestral susceptible genetic pool. PLoS One. 2010;5(4):e10034.

18. Jeon BC, Jeong SH, Bae IK, Kwon SB, Lee K, Young D, et al. Investigation of a nosocomial outbreak of imipenem-resistant Acinetobacter baumannii producing the OXA-23 beta-lactamase in korea. J Clin Microbiol. 2005;43(5):2241-5.

19. Dalla-Costa LM, Coelho JM, Souza HA, Castro ME, Stier CJ, Bragag nolo KL, et al. Outbreak of carbapenem-resistant Acinetobacter baumannii producing the OXA-23 enzyme in Curitiba, Brazil. $J$ Clin Microbiol. 2003;41(7):3403-6.

20. Hauck Y, Soler C, Jault P, Merens A, Gerome P, Nab CM, et al. Diversity of Acinetobacter baumannii in four French military hospitals, as assessed by multiple locus variable number of tandem repeats analysis. PLoS One. 2012;7(9):e44597.

21. Peymani A, Farajnia S, Nahaei MR, Sohrabi N, Abbasi L, Ansarin K, et al. Prevalence of class 1 integron among multidrug-resistant Acinetobacter baumannii in Tabriz, northwest of Iran. Pol J Microbiol. 2012;61(1):57-60.

22. Japoni S, Japoni A, Farshad S, Ali AA, Jamalidoust M. Association between existence of integrons and multi-drug resistance in Acinetobacter isolated from patients in southern Iran. Pol J Microbiol. 2011;60(2):163-8. 